Nuntius Antiquus, Belo Horizonte, v. 13, n. 2, p. 215-224, 2017

\title{
Il labirinto e l'artista. La sfinge e la statua nelle Lezioni di Estetica di G.W.F. Hegel
}

\section{O labirinto e o artista. A esfinge e a estátua nos Cursos de Estética de G.W.F. Hegel}

\author{
Giorgia Cecchinato \\ Departamento de Filosofia \\ Universidade Federal de Minas Gerais, Belo Horizonte, Minas Gerais / Brasil \\ giorgia.cecchinato@gmail.com
}

\begin{abstract}
L'obiettivo del testo è quello di presentare la lettura che G.W.F. Hegel (17701831) offre dell'arte dell'antico Egitto, in particolare delle piramidi e della sfinge. I colossali monumenti funerari e il mostro mitológico sarebbero, secondo l'interpretazione di Hegel, espressioni dello spirito e del popolo egizio, della sua cultura, della sua, della religione e del modo di vivere e sentire. In un secondo passo analizzeremo come Hegel usa il mito dell'enigma della sfinge per esemplificare la "superiorità" razionale dei greci in relazione all'antico Egitto. Si vedrà come, in ultima analisi, l'arte greca non è solo l'espressione di una luminosa razionalità conciliata con sé stessa.

Parole chiave: piramidi; sfinge; scultura; Hegel; Edipo.

Resumo: O objetivo do texto é o de, primeiramente, apresentar a leitura que G.W.F. Hegel (1770-1831) faz da arte do Antigo Egito, em particular das pirâmides e da esfinge. Os colossais monumentos funerários e a imagem mitológica seriam, conforme a interpretação do filósofo alemão, expressões do espírito e do povo egípcio, da sua cultura, da religião e do modo de viver e sentir. Em segundo lugar, analisar-se-á como Hegel usa o mito do enigma posto pela esfinge e resolvido por Édipo para exemplificar a "superioridade" racional dos gregos, em particular da arte grega em relação à do Antigo Egito. Ver-se-á como, em última análise, a arte grega também não é apenas expressão de uma luminosa racionalidade conciliada consigo mesma.
\end{abstract}

Palavras-chaves: pirâmides; esfinge; escultura; Hegel; Édipo. 


\section{Introduzione}

La teoria dell'arte di Hegel si basa sulla concezione dell'arte come espressione sensibile dell'idea, o in altre parole, del divino o della verità. In questa polarità tra mezzo di espressione sensibile e contenuto spirituale risiede il carattere paradossale dell'arte.

Nei suoi corsi di estetica, offerti all'Università di Berlino tra il 1820 e il 1829, il concetto di arte è pensato come paradigma del processo di idealizzazione progressivo della natura da parte dello spirito e, di modo corrispondente, come momento di sviluppo e attuazione del principio di libertà soggettiva.

Quando si parla di processo di idealizzazione della natura s'intende il processo con cui qualcosa di semplicemente naturale diventa capace di esprimere un contenuto razionale, per esempio una pietra che diventa un simbolo religioso o la semplice luce del giorno che diventa simbolo del divino. Nell'arte il processo di emancipazione dalla natura, come processo di idealizzazione passa per gradi diversi, dalla religione artistica, che semplicemente "trova" nella natura un oggetto che divento simbolo di un contenuto spirituale, Hegel offre come esempi, la luce o ad alcune pietre o cristalli, all'arte vera e propria che usa elementi naturali, pensiamo per esempio ai pigmenti di colore, per ricreare artificialmente effetti di luce, o l'uso del linguaggio per creare la poesia che per Hegel è la forma di arte suprema. L'idealizzazione come processo spirituale è anche, allo stesso tempo, il progressivo affermarsi di una libertà universale, come forma concreta di ragione che si esprime storicamente. Nel mondo arcaico, per esempio, nelle grandi civiltà orientali, la libertà era solo del despota ed era una libertà assoluta, perché non limitata dalla libertà altrui, tuttavia, non era una libertà completa perché il despota non riconosceva e non dava alcun valore a chi invece lo riconosceva come libero, cioè ai suoi sudditi. Nel mondo greco, nella Atene democratica, senza dubbio la reciprocità del riconoscimento tra i cittadini ha permesso il raggiungimento di un livello di libertà autentico, tuttavia esso non è universale perché limitato ai cittadini maschi, nati liberi. Il principio della libertà soggettiva è stato affermato e universalizzato, sempre secondo Hegel, dalla religione cristiana attraverso la morte e resurrezione di 
Cristo. La morte dell'uomo-Dio lancia storicamente, secondo Hegel, un messaggio universale di libertà e di uguaglianza che l'Europa raccoglie e sviluppa religiosamente, politicamente, e concettualmente, con Lutero, con la rivoluzione francese e con la filosofia di Kant rispettivamente. A questi tre momenti dello sviluppo della libertà corrispondono le tre articolazioni fondamentali del sistema dell'arte in Hegel: l'arte simbolica, che comprende l'arte delle antiche civiltà orientali; l'arte classica, ovvero l'arte del mondo greco, e l'arte romantica, così Hegel chiama l'arte cristiana che comprende l'arte dei primi secoli dopo cristo e che arriva sino al secolo XIX. Lo sviluppo dell'arte dunque, abbiamo visto, procede parallelamente all'articolarsi della dialettica natura-idealità e di quella della libertà del soggetto in relazione alla realtà oggettiva, politica e storica.

L'incontro della sfinge e di Edipo è localizzato proprio nel passaggio tra il mondo misterioso e grandioso dell'arte egizia, che Hegel intende come momento più alto dell'arte simbolica, e la "bella e luminosa eticità greca" espressa dall'arte classica. Nel mondo greco i cittadini sono coscienti della propria libertà e si riconoscono tra pari, l'arte riflette questa armonia e pienezza tra l'uomo e il suo mondo così come il livello superiore di libertà e di emancipazione dalla natura.

\section{Apollineo e dionisiaco in Hegel}

È così che la sfinge sorge nel mito greco, che possiamo nuovamente interpretare simbolicamente, come il mostro che propone l'enigma. La sfinge poneva la nota domande enigmatica: chi è che al mattino cammina con quattro zampe, nel pomeriggio con due e di notte con tre? Edipo trovò la semplice parola disvelatrice, l'uomo, e la sfinge cadde dalla rupe. La decifrazione del simbolo consiste nel significato esistente in sé e per sé nello spirito, come la famosa inscrizione greca intima all'uomo: conosci te stesso! La luce della coscienza è la chiarezza che lascia illuminare in modo chiaro il suo contenuto concreto attraverso la forma 
adeguata che gli appartiene e che espone, nella sua esistenza, solo a sé stessa. (HEGEL, 2000, vol II, p. 85. Trad. minha)

La soluzione dell'enigma posto dalla sfinge sta a significare il trionfo del carattere razionale della Grecia antica sulle civiltà antiche orientali, la conquista da parte del soggetto di una maggiore coscienza di sé e della propria libertà. Di modo corrispondente, l'arte greca, in questo caso la tragedia, incarnata da Edipo, mostra la sua superiorità rispetto alle grandiose e mostruose invenzioni mitologiche dell'Egitto.

Tuttavia, vorrei mostrare che il carattere paradossale dell'arte, così come Hegel la intende, ovvero come espressione sensibile di un contenuto spirituale, permane anche all'interno dell'arte greca e propriamente in relazione a quella che Hegel individua come la forma compiuta dell'arte greca: la scultura. Questo significa che, anche se nella scultura si esprimono, in modo eminente, la congiunzione del divino e dell'umano nella forma umana, propria del politeismo greco, anche se per mezzo equilibrio e della proporzione dei corpi si esteriorizza una visione razionale, potremmo dire "apollinea" del mondo, lo spirito che esprime nell'arte non può non trarre con sé un elemento oscuro, misterioso, "dionisiaco", che resiste alla luminosità razionale, come nascosto in un labirinto segreto, qualcosa che stona con la presunta perfetta congiunzione di materiale e spirituale che secondo Hegel è la caratteristica peculiare dell'arte classica.

Questa tensione mai risolta tra spirito e materia nell'arte non significa che non sia possibile pensare ad un progresso nel livello di libertà nell'arte o ad una sempre maggiore emancipazione del soggetto da modelli culturali e tradizioni, tuttavia impedisce di pensare ad un processo lineare e luminoso di sviluppo dell'arte sino ad arrivare alla cosiddetta "morte dell'arte". S'intende per "morte dell'arte" una teoria che prende le mosse da alcune affermazioni presenti nelle Lezioni di Estetica di Hegel, secondo le quali, l'arte sarebbe per lui e per i suoi contemporanei,q ualcosa di passato in quanto, diventando sempre più soggettiva, esaurirebbe il ruolo sociale-politico-culturale che aveva avuto per secoli. In sostanza l'estrema razionalizzazione dell'arte e la sua soggettivazione porterebbero alla perdita dell'oggettività propria 
dell'arte, in quanto produttrice di oggetti che si radicano in un mondo e che lo esprimono di maniera peculiare. L'arte, svuotata della sua funzione de esprimere sensibilmente in modo adeguato un contenuto spirituale, potrebbe dunque esprimersi adeguatamente alle esigenze del mondo moderno come riflessione sull' arte: come critica. Ridimensionare il carattere di perfetto equilibrio, o meglio, evidenziarne le crepe e le oscurità contribuisce anche a ridurre la portata della tesi della morte dell'arte, intesa come svuotamento epocale della funzione etica, culturale, sociale dell'arte in relazione al mezzo sensibile con cui si esprime, perciò diventano necessari un discorso sull'arte e una critica di arte. L'arte, pensata come paradosso di una soggettività spirituale che si esprime e di una cosa, un oggetto, un materiale, che la esprime permetterebbe di comprendere l'arte come qualcosa che accetta la propria morte come possibilità, che si espone alla possibilità di non essere compresa, inclusa e che anzi, vive solo grazie alle possibilità sempre nuove che la creatività soggettiva le infonde e solo grazie a questo è spirituale. I testi di Hegel permettono di sfuggire ad un'interpretazione rigida dell'equilibrio dell'arte Greca, come possibilità massima di espressione artistica, e mostrano che questa ha in sé un momento che non si lascia totalmente esteriorizzare.

Un'altra conseguenza, a mio modo di vedere, molto feconda di questo modo di interpretare la teoria dell'arte hegeliana, è che permette di moderare la visione di Hegel come un incallito classicista. Senza dubbio a partire dai testi, già dalle riflessioni giovanili, la cultura greca, espressa nell'arte, la tragedia come paradigma delle più alte contraddizioni dello spirito suscitano in Hegel, lettore e ammiratore di Winckelmann, è necessario ricordarlo, grande ammirazione, ma dare troppa enfasi alla centralità del bello greco nella teoria dell'arte di Hegel, rappresenterebbe un errore di prospettiva. Per evitarlo dunque vediamo come si svolge il passaggio dall'arte simbolica all'arte classica.

\section{La piramide e la sfinge}

Il momento il cui il naturale e il sensibile si mostrano come qualcosa di superato è il momento in cui l'arte propriamente detta nasce. 
Proprio nell'antico Egitto comincia il processo di idealizzazione della natura, ovvero il processo di rappresentazione spirituale per mezzo della natura, ma questa forma di rappresentazione è in qualche modo indiretta, poiché e rimane esteriore tanto alla natura, propriamente detta che allo spirito, che aspira ad una rappresentazione più adeguata. Per esempio gli egiziani adoravano dei con sembianza animale, dove il carattere animale del dio rappresentava una sua caratteristica spirituale. Ma gli antichi abitanti della Valle del Nilo andarono ben oltre questa rappresentazione di un contenuto spirituale, essi sono riusciti a negare nell'arte il carattere che è proprio della natura: la vita. Hanno così raggiunto una rappresentazione del carattere più proprio dello spirito, ovvero il sua essere negazione di tutto ciò che è immediato e naturale. Tuttavia il contenuto spirituale che nell'arte egiziana si impone immediatamente come ciò che nega la vita, è ancora solo negatività immediata, ancora solo come negatività naturale, è morte.

Questo livello immediato di negazione è inteso come ciò in cui consiste il significato assoluto e divino, il contenuto spirituale dell'arte. Nella mitologia egizia il dio riceve per la prima volta un destino umano e naturale: è chiamato ad affrontare la morte. Perciò la morte diventa momento della vita del dio-faraone, la quale permane sensibile, naturale e immediata. Di conseguenza il modo con cui la cultura egizia tentò di rendere immortali i propri dei faraoni non si discosta dal piano materiale e sensibile, essi perfezionarono infatti una tecnica per rendere eterno il corpo sensibile: la mummificazione.

Essi ebbero l'intuizione di de preservare ciò che era senza vita tanto nella sua esitsenza esteriore e interiore, quanto nella sua rappresentazione, e con questo realizzarono la transizione della coscienza i direzione della sua liberazione, benché siano arrivati appena ala soglia del regno della libertà. (HEGEL, 2000, vol II, p. 78. Trad. minha)

La spiritualità è appena presentita e intuita nella coscienza della vita e della morte del dio, nel processo con cui egli è mummificato, ovvero reso eterno e nelle opere che custodiscono questa immortalità, le piramidi. Questi monumenti funerari colossali, sono molto più che 
semplici tombe, ma d'altra parte, dal momento che la morte del dio è vista come assoluta, come ciò che abbraccia e conferisce significato alla vita, l'arte e la mitologia egizie hanno come proprio tema il processo circolare della vita come passaggio costante dalla vita alla morte e dalla morte alla vita. L'arte egiziana interiorizza questo processo che perde così il suo carattere sensibile e puramente naturale, si allontana dall'ambito di ciò che è familiare e abituale e produce opere che appaiono come apparizioni maestose ricoperte di mistero o nascoste e custodite nell'oscurità dei templi, protette da labirinti. Se da un lato questa forma di arte mostra un carattere di spiritualità maggiore, rispetto all'arte di altre civiltà antiche, dall'altro essa si trova comunque ancora imprigionata entro i limiti di una materialità naturale. Tutte le opere dell'arte egizia offrono un esempio di questa duplicità propria dell'arte simbolica, un esempio ancora oggi visibile sono le piramidi: da un lato esse si ergono nel sole cocente del deserto e esprimono la semplice circolarità della vita, retta dalla regolarità del ciclo solare, dall'altra rappresentano la venerazione della morte e dell'oscurità della terra. Esse nascondono labirinti e tunnel segreti che allontanano, e rendono misterioso il fatto, in sé solo naturale, della morte, e rendono eterno il dio in esse custodito. Nella loro forza e grandiosità proclamano la vittoria contro il tempo e contro la morte, la quale resta custodita e protetta dal labirinto.

La sfinge rappresenta un altro esempio di questa duplicità simbolica. Questa figura grottesca che mescola in sé l'umano e l'animale esprime il desiderio e l'esigenza dello spirito di essere liberato dalla natura, ma continua a rimanerne essenzialmente prigioniera. Nelle sue lezioni di filosofia della storia Hegel la descrive come segue: "Si può considerare la sfinge come simbolo dello spirito egizio: la testa umana che si erge sul corpo animale rappresenta lo spirito nel suo albore, come se si stesse appena elevando sopra il naturale per strapparselo di dosso e guardarsi intorno già libera, senza però liberarsi completamente dalla prigione".

Edipo libera la sfinge decretandone la morte. Egli rappresenta un altro popolo, un'altra mentalità. 


\section{La luminosità dell'arte greca $\mathrm{e}$ il labirinto interiorizzato}

Nell'arte greca cambia la relazione tra contenuto spirituale e opera perché cambiano il ruolo e la coscienza del soggetto non solo nell'arte ma in generale in tutte le espressioni dello spirito, nella società e nelle forme di sapere. Questo comporta una relazione diversa alla realtà oggettiva: la società greca era organizzata nella struttura politica della città-stato, la polis. Il soggetto è cittadino della polis e si riconosce immediatamente come libero. Vita pubblica e individualità non si distinguono, sostanza ética e soggetto sembrano vivere in un equilibrio perfetto senza nulla che minacci questa armonia. Ciononostante all'interno di questa vita libera e pregna di spiritualità nascerà la necessità di una libertà superiore che non sia solo libertà del cittadino della polis in quanto cittadino, ma di tutti gli uomini in quanto uomini. Nel suo momento culminante questa brillante civiltà unisce in modo armonico il mondo politico e sociale, da una parte, e il soggetto dall'altra, così come l'umano e il divino. Nella produzione artistica, il materiale costituisce l'elemento naturale, la materia. Nell'arte greca la materia è completamente compenetrata dallo spirito, al punto che l'apparenza sensibile è espressione perfetta dello spirito. Per questo la statua che rappresenta il dio-uomo è adorata come se il dio fosse l'opera stessa. Questo perfetto corrispondersi di forma e contenuto è quello che Hegel chiama "ideale del bello classico" incarnato dalla scultura. Tanto nelle Lezioni di Estetica, quanto nell'Enciclopedia delle scienze filosofiche in compendio Hegel enfatizza l'equilibrio della civiltà greca che risplende nello sua opere d'arte e particolarmente nel candore delle sua statue, tuttavia, in altre opere, come nella Fenomenologia dello Spirito per esempio, Hegel non tratta tanto della statua, né dell'essenza del bello dell'arte come compenetrazione perfetta tra forma e contenuto, sottolinea invece un aspetto che in qualche modo lo avvicina al labirinto in cui è custodito il dio egizio. La Fenomenologia sottolinea che l'opera è fatta da mani umane. Non si tratta di un elemento di scarsa importanza, tuttavia esso tende a restare in secondo piano rispetto all' aspetto per cui l'arte è rivelazione dello spirito. La tragedia, che Edipo rappresenta, è la forma di arte che annuncia la fine della "bella eticità greca".Hegel attribuisce un'importanza enorme alla tragedia, proprio nella tragedia, 
sotto la maschera dell'attore farà capolino il principio della soggettività che distruggerà l'equilibrio di cittadino e polis. La tragedia inoltre, rappresentando un potere più profondo e poderoso di quello degli dei, non può non portare allo spopolamento dell'Olimpo, ossia all'impossibilità di riconoscersi nelle divinità antropomorfiche del mondo classico. La tragedia annuncia la necessità da parte del soggetto libero di riconoscersi in un principio di più universale.

Tuttavia non è solo nella tragedia che si annuncia un principio spirituale che porterà l'arte greca e la civiltà che essa esprime a dissolversi, ma anche nella scultura che sembra a prime vista essere il momento più espressivo e perfetto di questa armonia. L'artista non riesce a mantenere una relazione rilassata con la propria opera. Mentre da un lato la statua rappresenta il tranquillo equilibrio tra l'opera e la società a cui è destinata, dall'altro lo stato d'animo dell'artista tradisce una situazione paradossale. Lui è teso perché non riesce a riconciliarsi con il contenuto dell'opera che lui stesso ha prodotto. Infatti, quando l'opera è adorata come il divino, l'artista senta un'incommensurabile distanza tra sé e il suo prodotto e non riesce a riconoscervisi. Tuttavia, di fronte alla devozione altrui della propria opera diventa cosciente che lui stesso è il maestro (der Meister), il produttore dell'opera e di conseguenza del divino che risplende per mezzo dell'opera (HEGEL, 2003, p.478-479)

Per il fatto che l'artista sa di aver creato lui stesso il dio rappresentato nell'opera, diventa lui stesso il custode del segreto della vita e della morte del dio, infatti questo dipende da lui. Il labirinto della piramide che custodiva il dio-faraone egizio è stato, per così dire, interiorizzato dall'artista e ora è nelle sue mani il mistero della vita e dell'eternità del dio. L'artista non ne ha consapevolezza precisa, infatti ha un presentimento, un tipo di coscienza oscura che si manifesta nel sentimento, una tensione. Il concetto di contenuto spiritale dell'opera si mostra dunque più complesso di quanto potrebbe essere apparso all'inizio, non si tratta solo del corrispondere tra cultura e arte, ma entra a far parte di questo contenuto spirituale qualcosa che non esce alla luce del giorno e che corrisponde al nucleo più propriamente spirituale dell'arte, ciò che riguarda la vita e la morte, l'umano e il divino. Nell'arte egizia 
questo nucleo oscuro dell' arte rimaneva custodito e protetto dal labirinto della piramide, nell'arte greca il labririnto prende la forma dell' interiorità dell'artista come enigma che nessun Edipo potrà svelare.

\section{Referências}

CECCHINATO, G. Traição e re-memoração. Reflexões sobre a experiência estética com base na Fenomenologia do Espírito. Discurso, USP, n. 42, p. 221-244, 2012.

FARINA, M. Critica, símbolo e storia in Hegel. La determinazione hegeliana dell'estetica. Pisa: Edizioni ETS Pisa, 2015.

GETHMANN-SIEFERT, A. Arte e religione, un effetto sinergetico nella questione illuminismo. In: IANNELLI, F. (Org.). Arte, religione e politica in Hegel. Pisa: Edizioni ETS, 2013.

GONÇALVES, M. C. O belo e o destino. Uma introdução à Filosofia de Hegel. São Paulo: Edições Loyola, 2001.

HEGEL, G. W. F. Cursos de Estética. Trad. port. Marco Aurélio Werle e Oliver Tolle. São Paulo: EDUSP, 2000. v. I-IV.

HEGEL, G. W. F. Fenomenologia do Espírito. Trad. Paulo Meneses, com a colaboração de Karl Efken e José Nogueira Machado, sj. Petrópolis: Vozes, 2003.

HEGEL, G. W. F. Vorlesungen über die Philosophie der Weltgeschchte. Band II-IV. Hamburg: Meiner Verlag, 1988.

IANNELLI, F. Bellezza ideale e disarmonia. In: FARINA F.; SIANI, A. L. (Org.). L'estetica di Hegel. Bologna: Il Mulino, 2014.

Recebido em 1 de novembro de 2017. Aprovado em 30 de novembro de 2017. 